\title{
Test Bed Design and Implementation for Biomedical Signal Analysis
}

\author{
Bhaskar Thakker \\ Department of Electronics \& \\ Communication Engineering \\ G H Patel College of \\ Engineering \& Technology \\ Vallabh Vidyanagar, India
}

\begin{abstract}
This document describes the fundamental requirements of a test bed along with its design and implementation for a biomedical signal acquisition and analysis. A test bed helps in acquiring the signal along with performing signal conditioning for amplification and filtration in simplified manner. Utilization of the Data Acquisition card (DAQ) along with LabView Signal Express software plays an important role in such a design. Such a test bed is designed and successfully tested in this paper for acquiring multi channel wrist pulse pressure signals. The importance of this test bed is concluded at the last. For any kind of long term biomedical signal acquisition, the test bed described here with implementation results gives guidelines for setting up the hardware with minimum efforts and greater accuracies.
\end{abstract}

\section{Keywords}

Test Bed Design; Test Bed Implementation; Data Acquisition Card (DAQ); LabView Signal Express; Pulse pressure sensor

\section{INTRODUCTION}

Biomedical signal processing and its analysis have got an attraction for the researchers all over the world since the advancement in the modern medical science. Awareness for the health care and longevity are increasing day by day which has led to the efforts from researchers in terms of understanding the behavior of human body in different abnormal health conditions by analyzing varieties of signals such as ECG, EEG, EMG, blood pressure signal etc. The research carried out for these signals in various abnormal health conditions have led to fruitful conclusions and that is why these signals are adopted by modern medical science for the health diagnosis.

There are certain requirements in terms of design and implementation of any biomedical signal acquisition system for the analysis of biomedical signals. The signals are always contaminated with noise like motion artifacts and power line interference [1]. Hence before the analysis of these signals, the noise is required to be eliminated. Usually the biomedical signal is also weak in strength due to the natural phenomenon in the body restricting to certain values for signal strength and hence the signal to noise ratio is also very poor. Due to these reasons, a primary requirement here is to amplify the weak information signal and filter out unwanted noise signals.

Motion artifacts are always of low frequencies in the range of few hertz near $0 \mathrm{~Hz}$ to around $2 \mathrm{~Hz}$. On the other hand the power line frequency noise are in integer multiples of $50 \mathrm{~Hz}$. Hence there may be requirements of either low pass filter, high pass filter or band pass filter depending upon the vital information present in specific band of frequencies for the given biomedical signal.

\section{SYSTEM REQUIREMENTS}

The most important point to be considered in the implementation of test bed for the biomedical signal is about the requirements of visualizing the signal during real time acquisition under the circumstances in which signal characteristics are dependent on several other parameters like placement of sensor, variable pressure required on the sensor etc. Few of such requirements are certainly present in case of EEG and ECG signals where the signal characteristics depend upon the placement of sensors. Hence before deciding final location of the sensor, the signal must be observed carefully while locating the sensor at different positions over the body. Such requirements demand for following points in real time manner, in the achievements of a test bed.

1. Acquisition of a biomedical signal

2. Amplification and filtering of the signal

3. Digitization of the signal

4. Display interface for the visualization of the signal

5. Long term storage of the signal

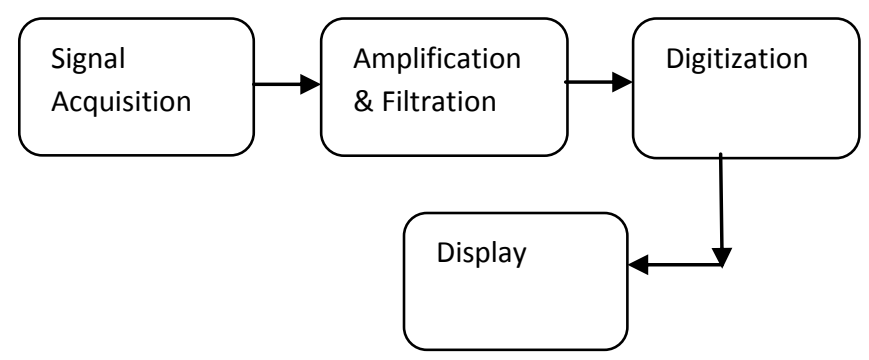

Fig 1: Block diagram for the Test bed implementation for Biomedical signal

The block diagram in Fig. 1 shows these requirements which mentions the selection of an appropriate sensor as well as analog front end circuit design for the signal acquisition. Analog filter will also be required to perform filtering in terms of Low Pass Filter, High Pass Filter or Band Pass Filter. Implementation of these analog filters may not be adequate especially for higher roll-off as the complexity of the circuit increases in such designs. Even the discrete circuit components like resistor, inductor and capacitors suffer from drift in their values over a period of time. This results in to the deviation of cut-off frequency as well as roll-off of the filter. Hence implementation of filtering task with the help of digital 
filter is a better choice in the implementation of test bed for Biomedical signal analysis.

In achieving such Test Bed, Data Acquisition Card and software like LabView Signal Express from National Instruments are ideal choice as described here.

\subsection{Data Acquisition Card (DAQ)}

Data Acquisition Card (DAQ) is an interface with the help of which any analog signal can be acquired, processed and then given to computer for the storage purpose. USB6009 as shown in Fig. 2(a) is such a utility provided by National Instruments with following features [2].

- 8 analog inputs (14-bit, $48 \mathrm{kS} / \mathrm{s}$ )

- 2 analog outputs (12-bit, $150 \mathrm{~S} / \mathrm{s}$ ); 12 digital I/O; 32-bit counter

- Bus-powered for high mobility; built-in signal connectivity

- Compatible with LabVIEW, LabWindows ${ }^{\mathrm{TM}} / \mathrm{CVI}$, and Measurement Studio for Visual Studio .NET
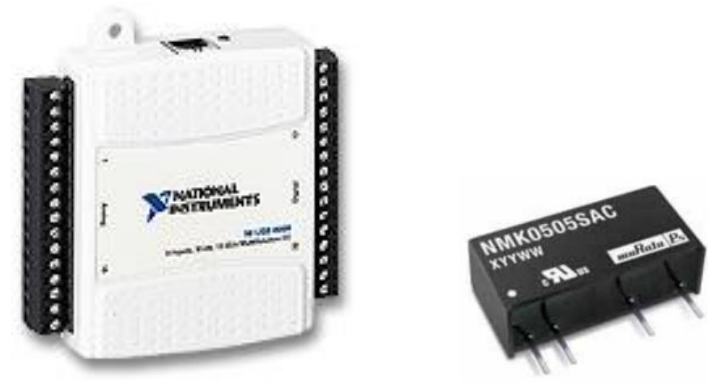

Fig 2: (a) Data Acquisition Card USB6009 (b) NMK0505SAC

This DAQ card can be utilized for data logging and portable measurements.

The instrumentation amplifier INA128 available in a signle surface mount package form is an ideal choice to minimize the space requirement in the implementation of analog front. INA128 provides noise rejection with $120 \mathrm{~dB}$ CMRR and hence is appropriate for the signal conditioning [3]. INA128 requires dual power supply for its operation. The computer is not having the provision for supplying power supply with +ve as well as -ve polarity. Hence an external battery needs to be interfaced for analog front end operations. If the data collection is to be carried out over longer period the choice of external batteries may not be an appropriate option. In place of batteries, DC to DC convertors provide better job here by providing dual power supply with the help of +5 Volt drawing from USB port. MK0505SAC as shown in Fig. 2(b) is such a DC to DC convertor which provides following features and can be utilized for the development of Test Bed for biomedical signal acquisition [4].

- $\quad 3 \mathrm{kVDC}$ isolation

- $\quad 5 \mathrm{~V}, 12 \mathrm{~V}, 15 \mathrm{~V} \& 24 \mathrm{~V}$ inputs

- $\quad 5 \mathrm{~V}, 9 \mathrm{~V}, 12 \mathrm{~V}, \& 15 \mathrm{~V}$ dual power output

- Fully encapsulated with toroidal magnetics

- No electrolytic or tantalum capacitors

\subsection{LabView Signal Express Software}

LabView Signal Express is an interactive and data-logging software for quickly acquiring, analyzing, and visualizing data from Data Acquisition Card (DAQ) without requiring any kind of program and by providing graphical environment [5]. The software communicates with the DAQ card through USB port and allows

1. To configure analog input channel

2. To amplify and digitize the analog signal

3. To configure the required Low Pass Filter, High Pass Filter or Band Pass Filter in terms of filter taps and cut off frequency

4. To store the data in file for long term recording and off line processing.

Fig. 3 shows a snap shot of LabView Signal Express software for data acquisition and Fig. 4 shows the hardware setup requirement for the test bed.

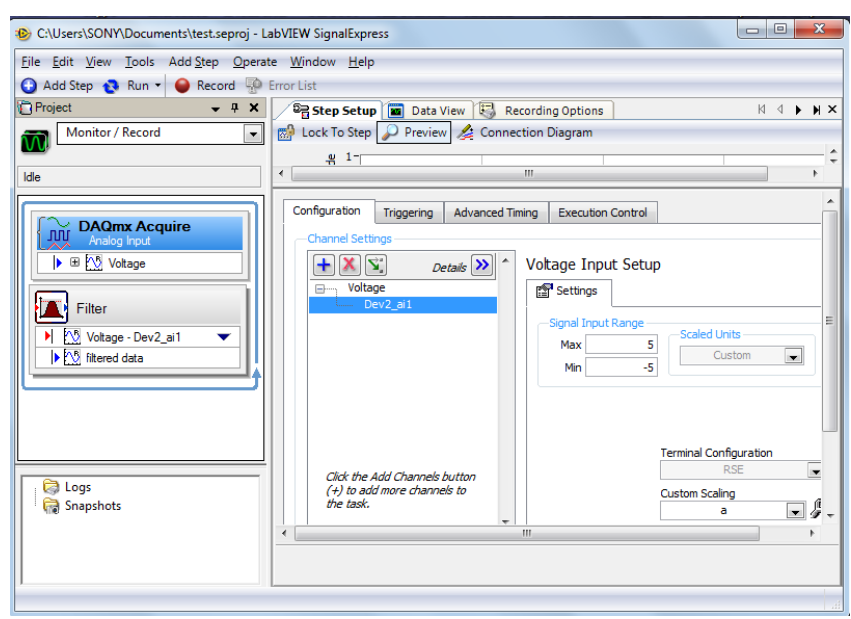

Fig 3: LabView Signal Express Software Environment

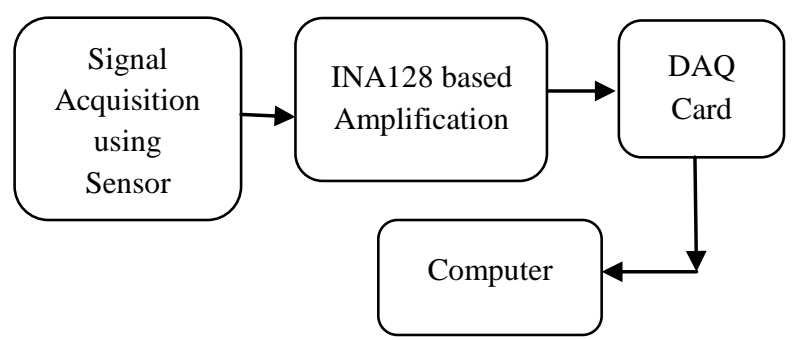

Fig 4: Test Bed for the Biomedical Signal Acquisition

\section{CASE STUDY}

As a case study the implementation of a Test Bed is discussed here for the acquisition, filtration and presentation of pulse pressure signal over radial artery in real time manner. In the pulse pressure signal acquisition as shown in Fig. 5, three fingers are required to be located over the radial artery at appropriate place which gives maximum pulse strength [6][7]. These signals are identified as Vata, Pitta and Kapha signals in the traditional approach of health diagnosis formally known as Ayurveda in Indian medicine. The pulse strength is dependent on the placement of the sensor, due to which an appropriate location over radial artery is required to be identified by monitoring the pulse signal simultaneously [8]. Since the pressure sensor is located on the body, the signal 
acquired is contaminated with power line frequency noise. Hence it is desired here to acquire the pulse pressure signal in real time manner, to filter the unwanted signals and to amplify the weak signal appropriately. At the same time the signal must be visualized so that the pressure sensor can be located appropriately over the radial artery. The other important characteristic of pulse signal is that the pulse strength varies according to the external applied pressure on the pulse point. Hence while applying external pressure on the pulse point, it is desired to verify the pulse strength simultaneously. This is only possible with the help of a unique combination of DAQ card with LabView Signal Express software. Here a tiny pressure sensor with make MPXM2053D working with piezoresistive principle has been utilized to show the results of real time test bed.
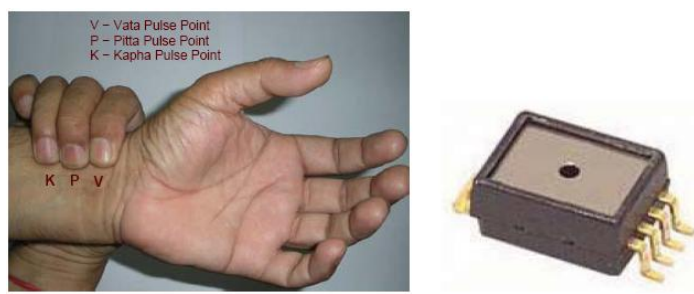

Fig 5: (a) Three Wrist Pulse Points

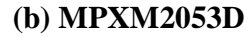

Pressure sensor MPXM2053D provides measurement of pressure signal with full scale pressure range of 0 - $50 \mathrm{KPa}(0-$ $375 \mathrm{mmHg}$ ) with $0.8 \mathrm{mV} / \mathrm{KPa} / \mathrm{Volt}$ sensitivity and $0.4 \%$ linearity [9]. The physical dimension of the sensor is $9.1 \mathrm{~mm}$ $X 7.1 \mathrm{~mm}$ in terms of length and width, which is almost similar to the surface area covered by fingertip surface and hence appropriate for the task of wrist pulse acquisition. The pin details are as shown in Table I.

Table 1. MPXM2053D Pin Details

\begin{tabular}{|c|c|l|}
\hline $\begin{array}{c}\text { Pin. } \\
\text { No. }\end{array}$ & $\begin{array}{c}\text { Pin } \\
\text { Symbol }\end{array}$ & \multicolumn{1}{|c|}{ Pin Detail } \\
\hline 1 & Gnd & Reference Supply Voltage \\
\hline 2 & Vout+ & Differential Positive Output \\
\hline 3 & Vs & Positive Supply Voltage \\
\hline 4 & Vout- & $\begin{array}{l}\text { Differential Negative } \\
\text { Output }\end{array}$ \\
\hline
\end{tabular}

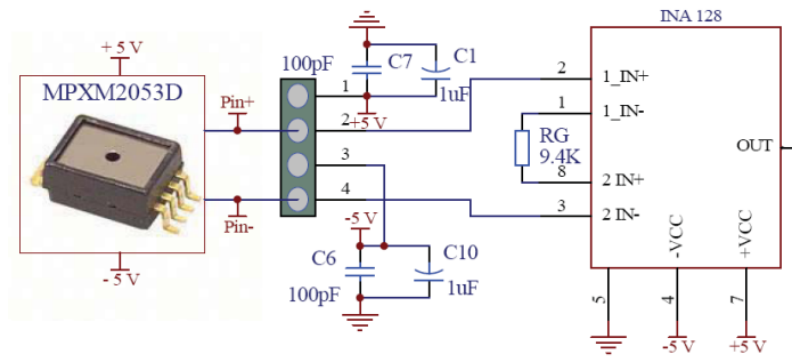

Fig 6: Instrumentation Amplifier designed for pulse signal acquisition

An Instrumentation amplifier INA128 as shown in Fig. 6 is utilized to amplify the weak signals from pressure sensor. The output of INA128 is given directly to DAQ card at one of the analog input channels.

\section{RESULTS}

The test bed for the acquisition of wrist pulse pressure signal is constructed as shown in Fig. 7. Through USB data cable DAQ card is interfaced with computer. The DAQ card is configured with the help of digital filter with $20 \mathrm{~Hz}$ cutoff frequency and 560 taps giving greater amount of roll-off to reject all the frequency components beyond $20 \mathrm{~Hz}$. The pulse pressure sensor is located over radial artery and filtered signal is observed in computer simultaneously to identify correct position of sensor based on the pulse strength.

USB Interface

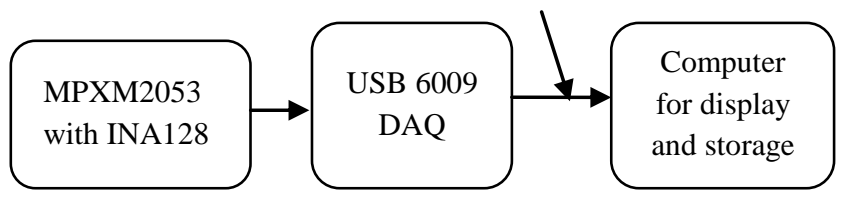

Fig 7: Test bed for pulse pressure signal acquisition

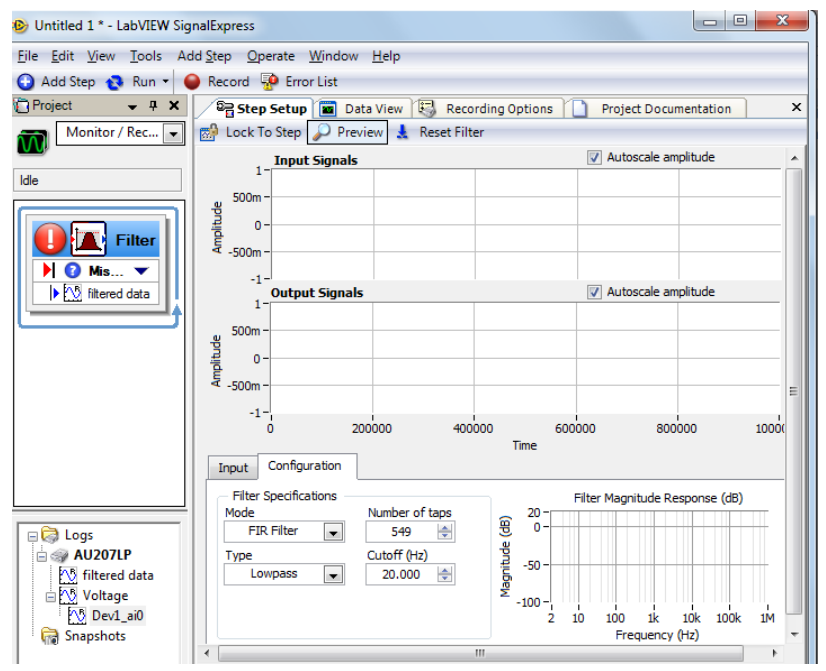

Fig 8: Filter design Environment with LabView Signal Express Software

Fig. 8 shows a snap shot of LabView signal Epress Software where the filter is configure for the acquired signal. The filter can be configured in terms of FIR or IIR as well as with required cut off frequency and number of taps resulting in greater flexibility for the user. Higher taps will lead to greater roll-off in the frequency response of filter.

\subsection{Single Channel Wrist Pulse Signal Acquisition}

Fig. 9 shows a single channel wrist pulse signal acquisition with the help of LabView Signal Express software. The first waveform is showing a signal as an output of digital filter implemented and the second signal shows the noisy signal given as input to the DAQ card. Both the signals are capture in real time manner. Here during pulse signal acquisition, static pressure is applied on the pulse point. Hence there exists static as well as dynamic pulse component both in the signal acquisition. The filtered signal can be recorded for long term duration. This recorded data can be generated in text format so that it can be further processed with MATLAB software. 


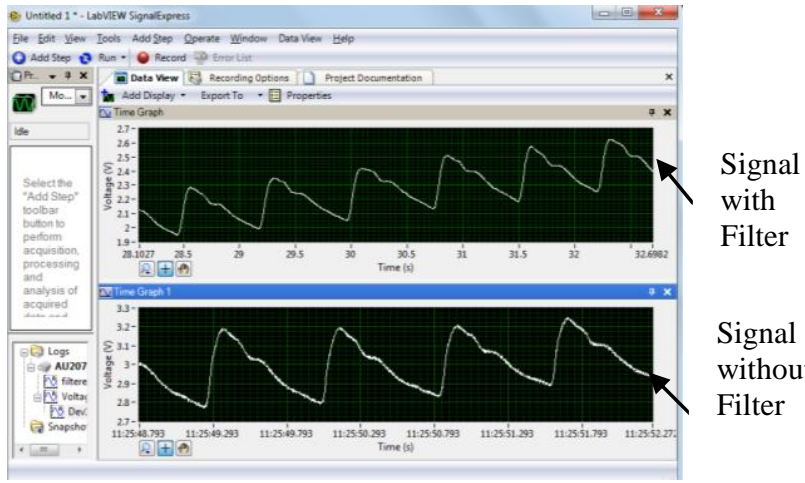

Fig 9: Single channel pulse recording with and without filter

\subsection{Multi Channel Wrist Pulse signal acquisition}

The DAQ card supports 8 analog channels to be simultaneously acquired with 48 Kilo Samples / sec. sampling rate together. All the three wrist pulse signals over radial artery identified as Vata, Pitta and Kapha are acquired and displayed on monitor screen as shown in Fig. 10. All the three signals can be recorded for long term and exported for off line processing as well as its power spectrum can also be calculated and displayed.

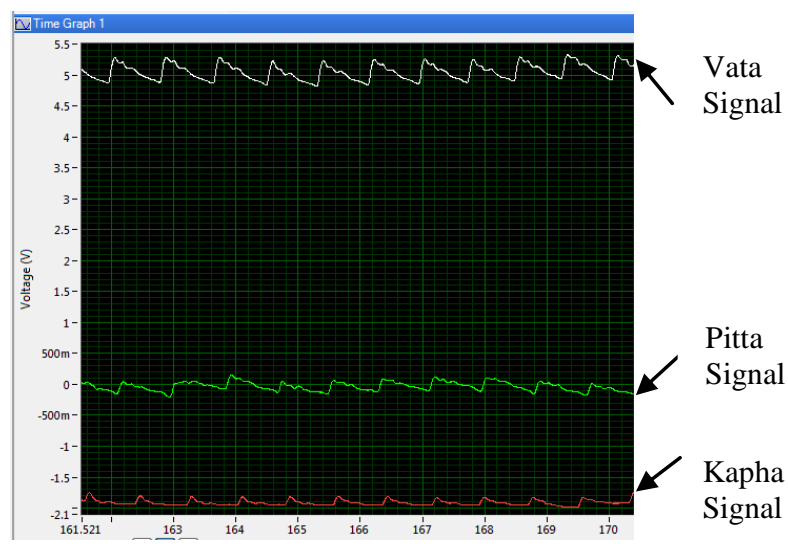

Fig 10: Multi channel pulse signal Acquisition

\section{CONCLUSION}

The test bed for a biomedical signal acquisition in real time manner is implemented considering amplification, filtration, visualization and long term recording of the signal. Utilization of the Data Acquisition Card along with LabView Signal Express software helps in achieving all the requirements for such a test bed. This test bed is useful for single as well as multi channel biomedical signals acquisition and analysis. As a case study, the test bed is successfully tested for three channel wrist pulse pressure signal. The test bed described and implemented in this work can be utilized for six lead ECG signals as well as EEG signals with 8 channels where placement of sensors is critical aspect for true signal acquisition. For any signal analysis task the primary requirement of acquisition for the signal in faithful manner is established with this test bed. Since DAQ card is of 14 bits resolution, it provides the digital output with 1 LSB as 0.3 $\mathrm{mVolts}$ at the rate of $48 \mathrm{Kilo}$ Samples/sec. This meets to any kind of requirement for the digitization of biomedical signals. For any kind of research work involving signal acquisition and analyzing the same, such test bed is a primary requirement and utilization of the same leads to the goals in short duration and greater accuracies.

\section{REFERENCES}

[1] A. Surve, P. Pandey, S. Pratapwar, V. Pandey, and N. Manigandan, "Enhancement of radial arterial pulse by spectral subtraction," 2004.

[2] “USB6009-Data Acquistion Card, National Instruments" http://sine.ni.com/nips/cds/view/p/lang/en/nid/201987

[3] "INA128 Data Sheet - Texas Instruments" http://www.ti.com/lit/gpn/ina128

[4] "NMK0505SC DC to DC converter" http://www.murataps.com/data/power/ncl/kdc nmk.pdf

[5] "National Instruments Signal Express Software" http://www.ni.com/labview/signalexpress/

[6] H. Rhyner, Ayurveda: the gentle health system, vol. 18 . Motilal Banarsidass Publ.,1998.

[7] S. Upadhyaya, Nadi Vijnana - Ancient Pulse Science. Chaukhamba Sanskrit Pratishthan, 2005.

[8] Bhaskar Thakker and Anoop Lal Vyas "Radial Pulse Analysis at Deep Pressure in Abnormal Health Conditions" IEEE International Conference on Biomedical Engineering and Informatics (BMEI 2010), ISBN: 978-1-4244-6496-8 October 2010 Yentai, China, pp. 1007-1010.

[9] "Pressure Sensor MPXM2053D from Freescale Semiconductor"

http://cache.freescale.com/files/sensors/doc/data_sheet/M PX2053.pdf 